\title{
Corrigendum: Structural characterization of human heparanase reveals insights into substrate recognition
}

Liang Wu, Cristina M Viola, Andrzej M Brzozowski \& Gideon J Davies

Nat. Struct. Mol. Biol. 22, 1016-1022 (2015); published online 16 November 2015; corrected after print 8 December 2015

In the version of this article initially published, there were errors in two figures. The schematic in Figure 4 a was mistakenly drawn with a $\beta 1 \rightarrow 3$ linkage between the -1 GlcUA and the +1 GlcNS(6S), rather than the correct $\beta 1 \rightarrow 4$ linkage. Supplementary Figure 1 incorrectly gave the names of the GlcNX monomers as $N$-acetyl- $\alpha$-D-glucuronic acid and $N$-sulfo- $\alpha$-D-glucuronic acid, rather than $N$-acetyl- $\alpha$-D-glucosamine and $N$-sulfo$\alpha$-D-glucosamine. These errors have been corrected in the HTML and PDF versions of the article. 